\title{
Oral hygiene - more than just brushing
}
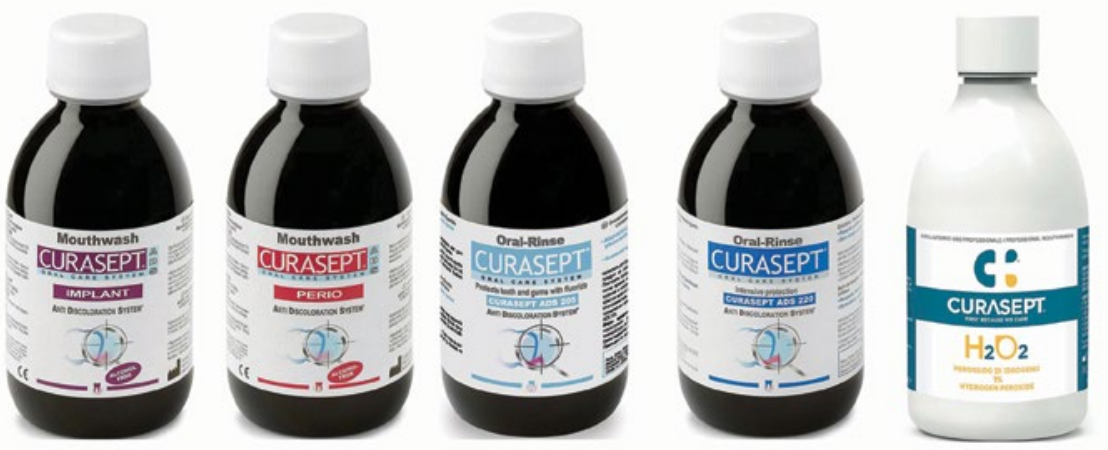

For patients to maintain optimal oral hygiene at home, it takes more than just brushing their teeth. Why not introduce them to the Curasept ADS range of mouth rinses to take their dental health to the next level?

Available from J\&S Davis, Curasept ADS products contain chlorhexidine for plaque fighting benefits, while being formulated to minimise the risk of staining and taste disturbance associated with other solutions. There are also targeted therapies like Curasept ADS Implant and Curasept ADS Perio, which include hyaluronic acid to accelerate healing and PVP-VA to combat future plaque accumulation.

To help your patients elevate their homecare dental routines, remind them it takes more than just brushing. Recommend Curasept from J\&S Davis.

For more information on the industryleading products available from J\&S Davis, visit www.js-davis.co.uk, call 01438747 344 or email jsdsales@js-davis.co.uk.

\section{For your endodontic referrals}

EndoCare's philosophy is ensuring that every patient gets the help they need, regardless of the complexity of the case.

With decades of experience behind them, the team are all trained to perform only the most up-to-date endodontic treatments and techniques, meaning that they can offer patients practically pain-free treatment and solve even the most complex cases.

When you refer to EndoCare you can rest assured that they will return your patients to your care with comprehensive details of the treatment they have received and EndoCare's suggested next steps.

Get a helping hand and give your patients the specialist care they need by contacting EndoCare today.

For further information call EndoCare on 02072240999 or visit www.endocare.co.uk.

\section{A unique dental implant solution}

Complex bone augmentation procedures are often stretched to their limits in cases of high-risk patients, poor compliance and extreme bone resorption. Zygoma dental implants offer both patients and clinicians an alternative if surgical intervention in the upper jaw is indicated.

The new SZ-75 handpiece has been developed by W\&H in collaboration with leading Zygoma dental implantologists. It is specifically designed to enable ultra-safe Zygomatic dental implant surgery and placement, with an innovative chucking system that makes this contra-angle handpiece the only one of its kind.

Zygoma implants are inserted into the zygomatic bone at a specific angle. It is crucial that the implant is accurately positioned to ensure success. The new W\&H SZ-75 handpiece makes Zygomatic implant procedures easier to perform, as the ergonomic angulation of the instrument facilitates optimal access to the treatment site. This enables the clinician to work for extended periods of time with minimum risk of strain or fatigue.

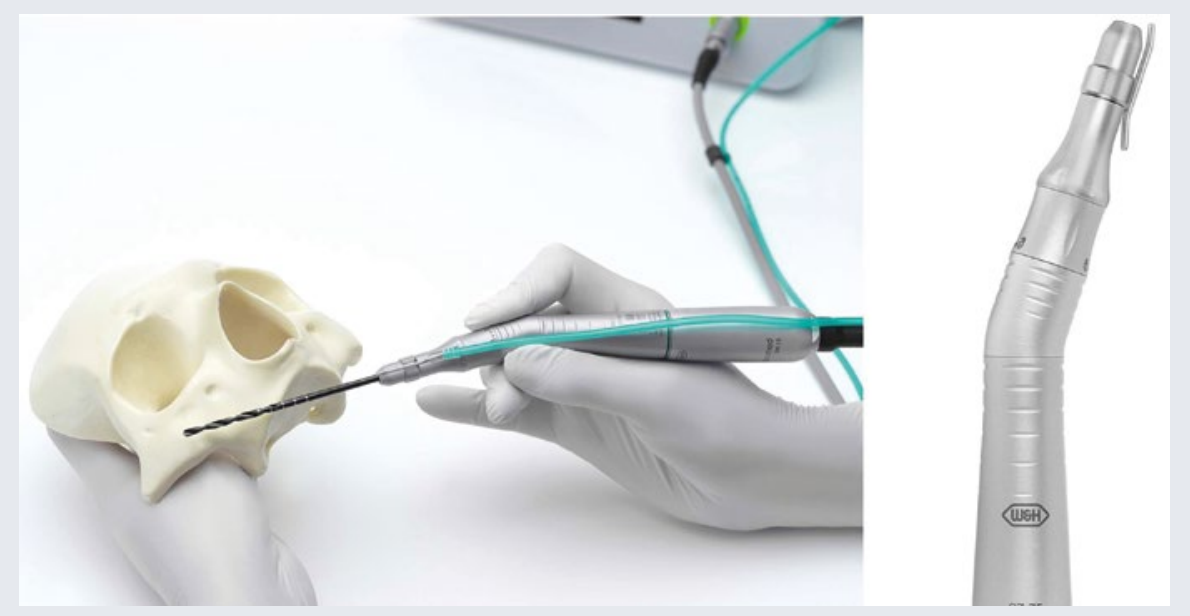

Conventional surgical handpieces do not always clutch Zygoma implant burs securely. The SZ-75 from W\&H is the world's first 20:1 contra-angle handpiece to combat this issue with a unique chucking system that is optimised for Zygomatic dental implant surgery. This innovative solution makes the SZ-75 ultra-safe to use, whilst ensuring highly accurate concentricity during application. In addition, the handpiece can be used with standard dental implant burs and applied universally in surgery.

The new W\&H Zygoma handpiece also features an external spray channel that expels coolant close to the tip of the instrument to ensure efficient cooling of the treatment site, thus eliminating the need for manual cooling.

To find out more visit www.wh.com/ en_uk, call 01727874990 or email office. uk@wh.com. 